Supplement of The Cryosphere, 15, 897-907, 2021 https://doi.org/10.5194/tc-15-897-2021-supplement (C) Author(s) 2021. This work is distributed under the Creative Commons Attribution 4.0 License.

(c) (i)

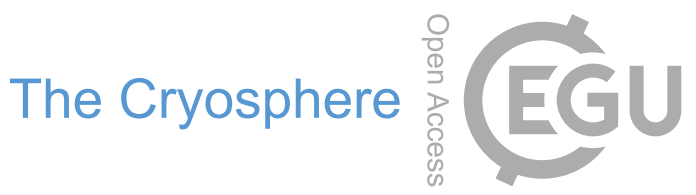

Supplement of

\title{
The cooling signature of basal crevasses in a hard-bedded region of the Greenland Ice Sheet
}

Ian E. McDowell et al.

Correspondence to: Ian E. McDowell (ian.mcdowell@ nevada.unr.edu)

The copyright of individual parts of the supplement might differ from the CC BY 4.0 License. 


\section{S1. Decay of thermal signal created during drilling compared to observed temperature changes}

An analytical model describing the thermal decay from linear heat sources in ice is obtained from the solution to a line source within an infinite media presented by Carslaw and Jaeger (1959):

$$
T(l, t)=\frac{Q}{4 \pi \kappa \rho C t} e^{-\frac{l^{2}}{4 \kappa t}}
$$

where $l$ is the linear distance away from the heat source, $t$ is the time that has elapsed, $Q$ is the amount of heat per unit length of the line source, $\kappa$ is the thermal diffusivity, $\rho$ is the density of the of the medium, and $C$ is the specific heat capacity. Table S1 reports the values for all constants used in this study.

Humphrey and Echelmeyer (1990) modified Eq. (S1) to estimate the temperature at time $t$ for sensors installed in a borehole using a hot water drill. Setting $l=0$ removes the exponential term from the equation. Humphrey and Echelmeyer (1990) also substituted thermal conductivity $(K)$ for $(\kappa \rho C)$ and added the term $T_{0}$ to account for the ambient temperature of the ice before being disturbed by drilling. This leads to equation 24 in Humphrey and Echelmeyer (1990):

$$
T(t)=T_{0}+\frac{Q}{4 \pi K t}
$$

Equation (S2) illustrates the asymptotic temperature decrease which is proportional to inverse time. The $Q$ term is amount of heat per unit length of the borehole and includes both sensible heat during drilling and latent heat from the water refreezing. The sensible heat required to raise the temperature of ice to its melting point is small compared to the large amount of latent heat required to melt the borehole. $Q$ is given by:

$$
Q=m_{w} L_{f}+m_{i} C \Delta T
$$

where the right-hand terms are the latent heat of refreezing in the borehole and the sensible heat to warm the melted ice up to $0{ }^{\circ} \mathrm{C}$. The mass of water in $1 \mathrm{~m}$ of the borehole is:

$$
m_{w}=\rho_{W} \pi r^{2} h
$$

Here $r$ is the radius of the borehole, which we assume equals $5 \mathrm{~cm}$ if the boreholes have approximately a $10 \mathrm{~cm}$

diameter. The mass of ice raised to its melting temperature is represented by $m_{i}$ and is found by solving Eq. (S4) but substituting the density of ice for the density of water.

We analyze the thermal behavior of a borehole to ensure that the rates of cooling we observe is not an artifact of residual borehole cooling. We find that after a year, the residual temperature changes of the borehole are well below sensor resolution; however, to avoid any potential contamination of both the static and temporal trend of the sensor temperature data from the thermal disturbance of installation, we ignore the first year of data in our analysis. 


\begin{tabular}{|l|c|c|c|}
\hline \multicolumn{1}{|c|}{ Table S1. Constants used in this study (Cuffey and Paterson, 2010) } \\
\hline \multicolumn{1}{|c|}{ Property } & Variable & Value & Units \\
\hline Density of ice & $\rho_{i}$ & 917 & $\mathrm{~kg} \mathrm{~m}^{-3}$ \\
\hline Density of water & $\rho_{w}$ & 1000 & $\mathrm{Kg} \mathrm{m}^{-3}$ \\
\hline Heat capacity of ice & $C$ & 2097 & $\mathrm{~J} \mathrm{~kg}^{-1} \mathrm{~K}^{-1}$ \\
\hline Latent heat of fusion & $L_{f}$ & $3.335 \times 10^{5}$ & $\mathrm{~J} \mathrm{~kg}^{-1}$ \\
\hline Thermal conductivity of ice & $K$ & 2.1 & $\mathrm{~W} \mathrm{~m}^{-1} \mathrm{~K}^{-1}$ \\
\hline Thermal diffusivity of ice & $\kappa$ & $1.09 \times 10^{-6}$ & $\mathrm{~m}^{2} \mathrm{~s}^{-1}$ \\
\hline Clausius-Clapeyron slope & $\gamma$ & $-7.4 \times 10^{-8}$ & ${ }^{\circ} \mathrm{C} \mathrm{Pa}^{-1}$ \\
\hline
\end{tabular}

\section{S2. Estimating the temporal change of temperature in a stepped digital record}

The lengths of our temperature records are restricted by the date of installation after the borehole was drilled, and

the date of the last data collection in July 2017. The time change of temperature is near the digital resolution of the temperature sensors, which can result in a stair stepping of the record over only a few steps and over the entire record (see Fig. S1 for an extreme example). Applying a linear regression to the raw data can lead to a biased estimate of the rate of change of temperature. As an example of this problem, we look at a full sensor record showing a decrease of approximately $0.1^{\circ} \mathrm{C}$ through time (Fig. S1).

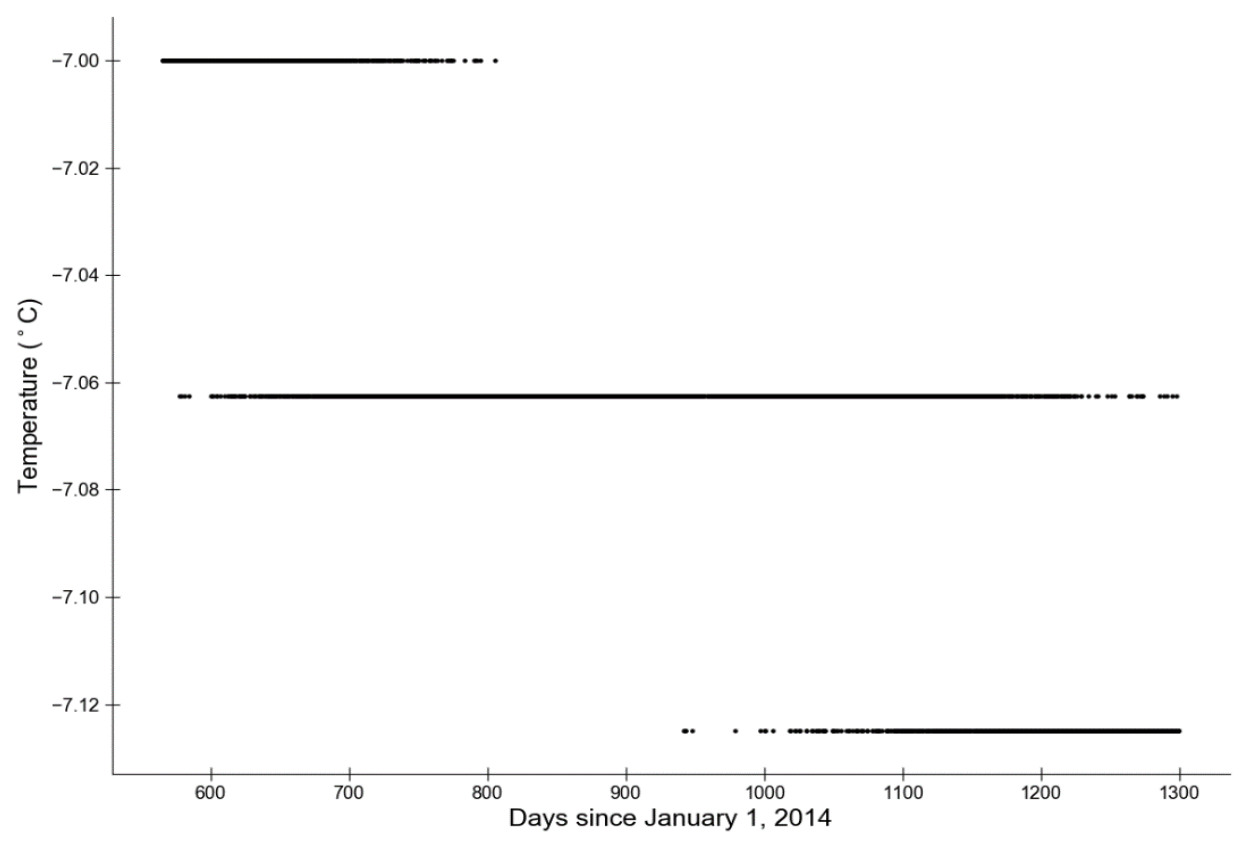

Figure S1. Temperatures (black dots) recorded over two years by sensor \#15, which is 200 meters above the bed in borehole 14SA. When plotted over time, the temperatures display characteristic stair-stepping pattern between resolution steps of the sensor. 
Both the first and last digital steps of this record have fewer entries recorded due to the restrictions of the sampling window. Our treatment of appending truncated data and performing a least-squares regression is illustrated in Fig. S2, which also shows the difference in derived rates of temperature change over time. Our method provides a more conservative estimate of the rate of temperature change over time than if we had we not padded the entries in the first and last resolution steps. In Fig. S2, the slope to the original line of best fit was $-5.3 \pm 0.05 \times 10^{-2}{ }^{\circ} \mathrm{C} \mathrm{yr}^{-1}$, while the slope of the line fit to the augmented data was $-4.4 \pm 0.02 \times 10^{-2}{ }^{\circ} \mathrm{C} \mathrm{yr}^{-1}$ (mean \pm st. error).

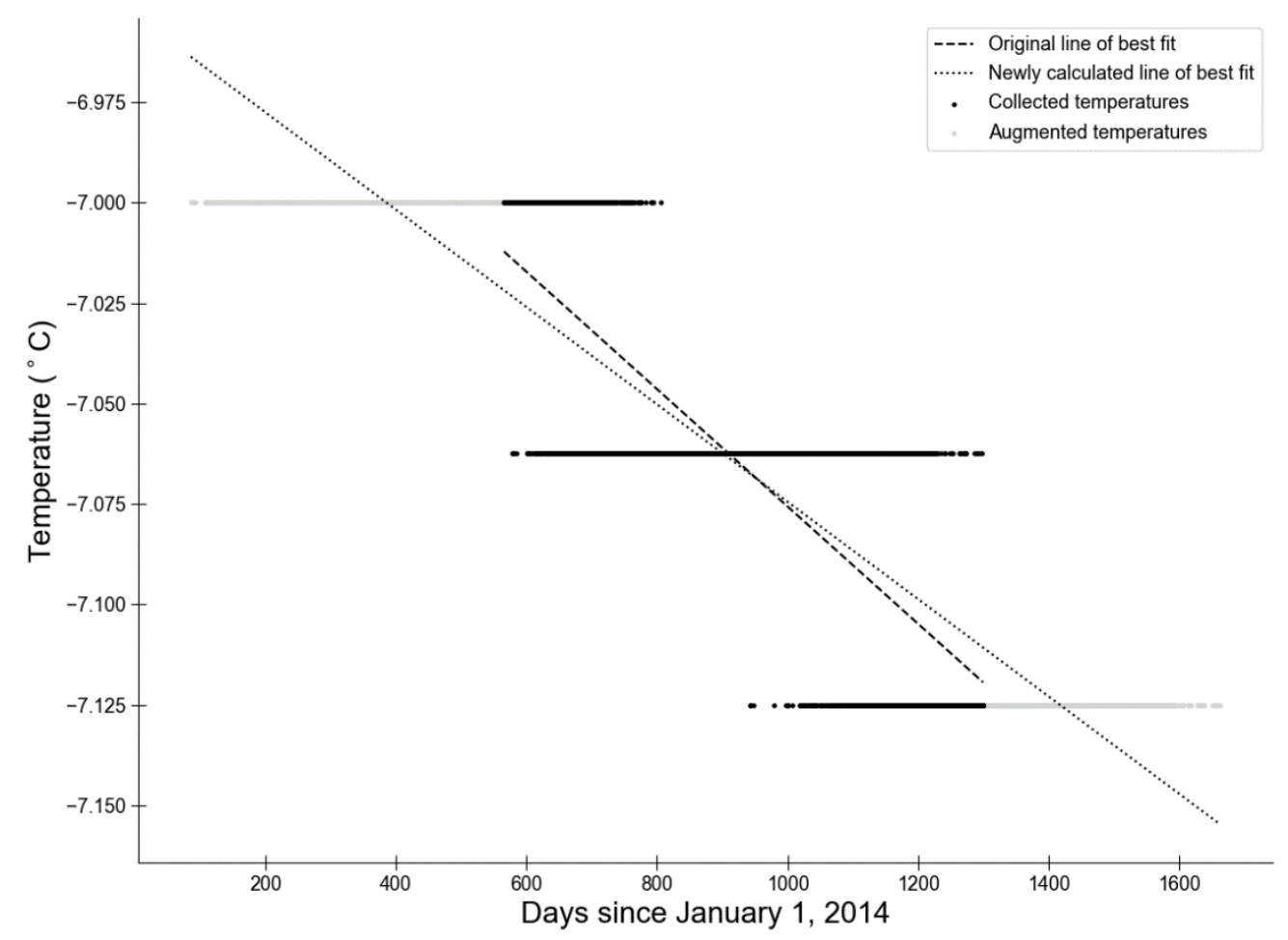

Figure S2. Temperatures (black dots) recorded over two years. The augmented records are shown with light grey points. The original least-squares line of best fit is shown with the black dashed line and the dotted line is the newly calculated trend using the augmented data. 


\section{S3. Modeling distributed cooling from multiple basal crevasses}

We model a field of 3 basal crevasses that are $1 \mathrm{~m}$ thick with a 1-D diffusion model in the direction perpendicular to the crevasses and at some height above the bed. Our finite difference model domain is $800 \mathrm{~m}$ wide,

45 covering 3 crevasses spaced $100 \mathrm{~m}$ apart. Initial ice temperature is $-4^{\circ} \mathrm{C}$, which is the average temperature in the lowest $200 \mathrm{~m}$ of the ice column from our borehole temperature measurements. To simulate three basal crevasses, 1 m wide, $0{ }^{\circ} \mathrm{C}$ nodes are located at 300,400 and $500 \mathrm{~m}$. Boundary nodal temperatures are fixed at $-4{ }^{\circ} \mathrm{C}$, while temperatures throughout the rest of the domain evolve following Eq. (2) for 30 years. The model duration is chosen based on our timescale analysis, which suggests that crevasses occurring 20 - 30 years ago could produce the 50 cooling rates in each borehole.

\section{References}

Carslaw, H. S. and Jaeger, J. C.: Conduction of Heat in Solids, 2nd ed., Clarendon Press., 1959.

Humphrey, N. and Echelmeyer, K.: Hot-water drilling and bore-hole closure in cold ice, J. Glaciol., 36(124), 287298, 1990. 